\title{
Field evaluation of an automated RDT reader and data management device for Plasmodium falciparum/Plasmodium vivax malaria in endemic areas of Colombia
}

Sócrates Herrera ${ }^{1,2^{*}}$, Andrés F Vallejo ${ }^{1,2}$, Juan P Quintero ${ }^{1,2}$, Myriam Arévalo-Herrera ${ }^{1,2,3}$, Marcela Cancino ${ }^{4}$ and Santiago Ferro ${ }^{4}$

\begin{abstract}
Background: Massive implementation of malaria diagnostics in low-resource countries is regarded as a pivotal strategy in control and elimination efforts. Although malaria rapid diagnostic tests (RDTs) are considered a viable alternative, there are still obstacles to the widespread implementation of this strategy, such as reporting constraints and lack of proper quality assurance of RDT-based programmes at point-of-care (POC).

Methods: A prospective cohort of patients, seeking routine care for febrile episodes at health centres in malaria-endemic areas of Colombia, was used to assess the diagnostic performance of a device based on smartphone technology (Deki Reader ${ }^{\mathrm{TM}}$, former codename "GenZero"), that assists users at POC to process RDTs. After informed consent, patients were enrolled into the study and blood samples were collected for thick blood smear (TBS) and RDT. The RDT results were interpreted by both visual inspection and Deki Reader device and concordance between visual and device interpretation was measured. Microscopy corrected by real-time polymerase chain reaction (PCR) and microscopy were "gold standard" tests to assess the diagnostic performance.

Results: In total, 1,807 patients were enrolled at seven health centres in malaria-endemic areas of Colombia. Thirty-three Plasmodium falciparum and 100 Plasmodium vivax cases were positive by corrected microscopy. Both sensitivity and specificity were $93.9 \%(95 \% \mathrm{Cl}$ 69.7-95.2) and $98.7 \%(95 \% \mathrm{Cl} 98.5-99.4)$ for $P$. falciparum, and 98.0\% (95\% Cl 90.3-98.9) and 97.9\% (95\% Cl 97.1-98.5) for P. vivax. Percentage concordance between visual and device interpretation of RDT was $98.5 \%$ and $99.0 \%$ for $P$. vivax and $P$. falciparum, respectively. The RDT, when compared to TBS, showed high sensitivity and specificity for $P$. falciparum in both visual and device interpretation, and good overall diagnostic performance for P. vivax. Comparison between PCR as gold standard and visual and device interpretation showed acceptable overall performance for both species.

Conclusions: The diagnostic performance of the Deki Reader was comparable to visual interpretation of RDTs (without significant differences) for both malaria species. Providing standardized automated interpretation of RDTs at $\mathrm{POC}$ in remote areas, in addition to almost real-time reporting of cases and enabling quality control would greatly benefit large-scale implementation of RDT-based malaria diagnostic programmes.
\end{abstract}

Keywords: DekiReader, Malaria rapid diagnostic test, $\mathrm{PCR}$, mHealth

\footnotetext{
* Correspondence: sherrera@inmuno.org

${ }^{1}$ Caucaseco Scientific Research Center, Cali, Colombia

2Latin American Center for Malaria Research, Cali, Colombia

Full list of author information is available at the end of the article
} 


\section{Background}

Malaria is transmitted in about 106 countries in tropical and subtropical regions where $\sim 3.3$ billion people, half of the world's population, are at risk of malaria transmission. The World Health Organization (WHO) estimates that over 219 million cases (uncertainty range of 154 to 289 million) and about 660,000 malaria deaths (uncertainty range of 490,000 to 836,000 ) occur worldwide every year [1], representing an enormous global social and economic burden. Clinical manifestations of the disease are similar to many other infectious diseases making clinical diagnosis alone imprecise and inaccurate. Confirmation of malaria, which depends on the detection of Plasmodium parasites in blood circulation, is not only critical for appropriate case management [2], but it is essential for epidemiological purposes and to enhance malaria control programmes.

Microscopic detection of Plasmodium species on thick blood smear (TBS) has been for decades the standard method for malaria diagnosis despite its field accuracy, errors in species identification and its operator-dependence. Although it is less expensive and easier to handle than other malaria diagnostic methods developed more recently, it is laborious and time consuming, and it requires electricity as well trained personnel, frequently not available in remote malaria-endemic areas. Recently, a number of techniques aimed at reducing the time of processing and some other constraints of TBS have been developed. Some of them include fluorescence stains but still require microscopes, others are based on detection of parasite nucleic acids in human blood circulation but require expensive and complex equipment, and others are based on the use of antibodies that recognize parasite components $[3,4]$. The methods based on parasite nucleic acid detection, have proven great sensitivity and specificity, however, they require significant infrastructure and training, and are significantly more expensive than TBS, therefore precluding expanded implementation in low-resource settings [5]. Attempts to simplify these methods are currently underway based on loop-mediated isothermal amplification (LAMP) which requires less sophisticated equipment, such as a water bath and ultraviolet light, which could be installed in more underserved regions, but still requires electricity [6].

Malaria diagnostic methods based on the use of monoclonal antibodies recognizing parasite antigens $[3,4]$ have been grouped under the name of malaria rapid diagnostic tests (RDT). These methods have shown great performance in term of sensitivity and specificity, although they still present limitations, such as batch-to-batch variation, and limited sensitivity at low parasitaemia levels $(<0.1 \%)$. Although these RDTs still require improvement, their apparent simplicity currently makes them attractive to provide diagnosis at all levels of the health care system, particularly in remote areas where health workers have limited supervision and training.
The reliability of RDT results is pivotal to ensure the safety of withholding anti-malarial treatment in testnegative patients [7-10]. As recommended by WHO, the possibility of diagnosis error using RDTs can be minimized through a careful selection process [11]. Important steps involved in the process of ensuring high quality of RDTs include among others, high diagnostic performance as assessed in product testing by the WHO and the Foundation for Innovative New Diagnostics (FIND) [12], proper storage of the test kits, adequate labelling, clear operation instructions, continuous on-the-job training (job aids), and automated interpretation to avoid inter-user variability. In addition, implementation of large-scale diagnostic programmes based on malaria RDTs requires the establishment of a comprehensive quality assurance (QA) strategy. This task can be particularly challenging considering that most RDTs are being used at point-of-care (POC) in health centres and dispensaries located in remote areas.

The performance of health professionals using RDTs can be improved through simple redesign of job aids $[7,8,13,14]$, as well as by QA programmes based on supervisory visits to assess their competence to correctly use and interpret RDTs results [15]. However, the sustainability of this type of QA programme is highly questionable due to costs of personnel, transportation and other expenses [16,17]. Currently available technologies such as cell-phone networks, could reduce costs and increase the efficiency as a supervisor could conduct QA activities to multiple facilities without the need to visit the sites, as shown in preliminary studies with both malaria and HIV RDT programmes [18-20].

The present report describes a field trial in malariaendemic areas of Colombia of a system that includes mobile, battery-operated devices based on smartphone technology that assists users at POC to process RDTs, performing automated interpretation, reducing notification time to a cloud-based database and allowing malaria case geo-location and remote QA activities. The main objective of the trial was to assess the concordance between automated interpretation of RDTs by the device and by visual inspection by laboratory experts.

\section{Methods \\ Study design and study sites}

The present study involved a prospective cohort of consecutive patients from August 2011 to January 2012 at seven sites in four towns located in malaria-endemic areas of Colombia (Figure 1): Tumaco and Buenaventura in the Pacific coast, and Monteria and Tierralta in the northwestern department of Córdoba. These four sites were selected based on their high malaria incidence and their reliable mobile phone connectivity. Patients were recruited at the main governmental malaria diagnosis facility, regional hospitals and Public Health Laboratory and at a diagnostic 


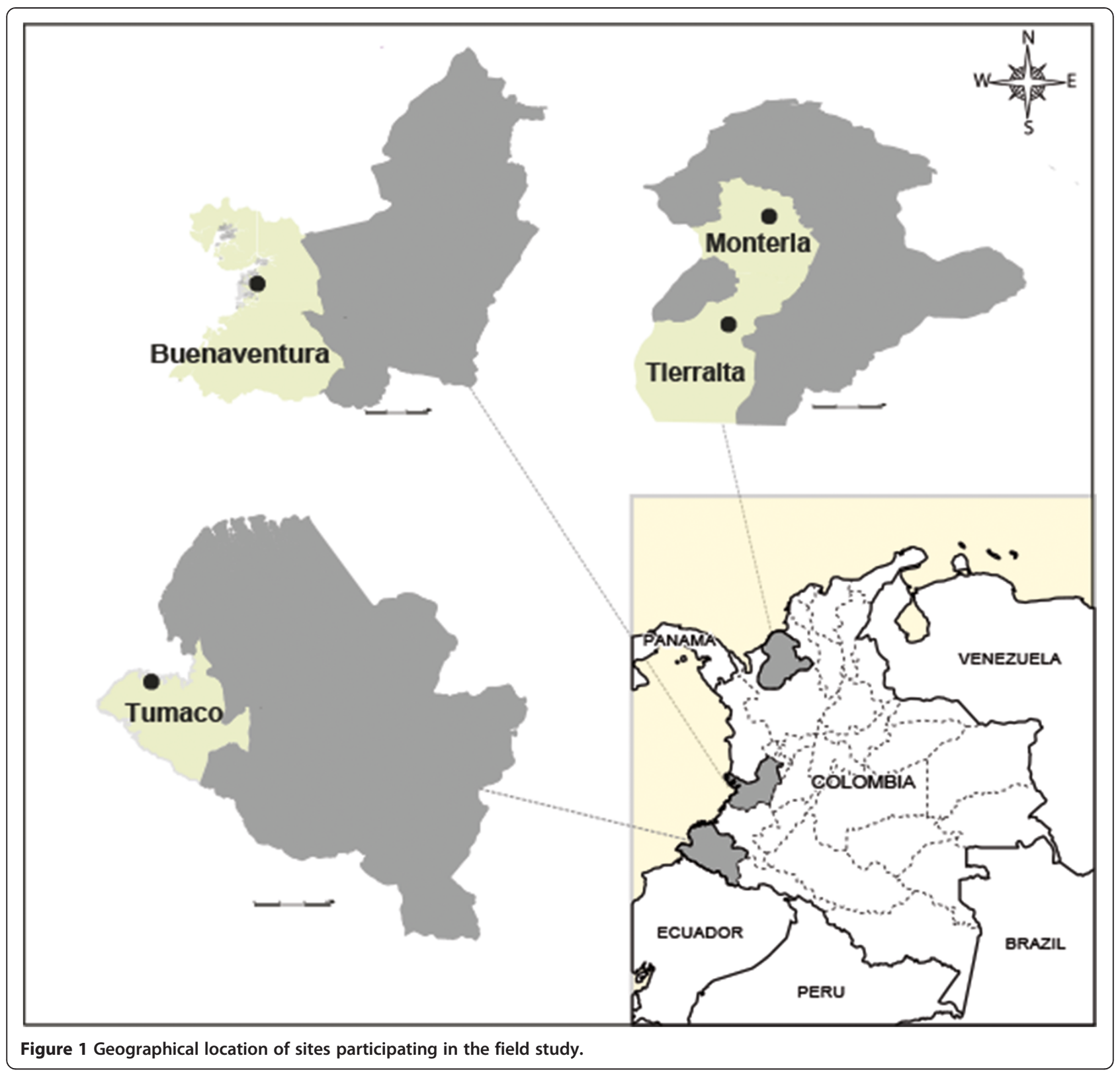

centre established by Caucaseco Scientific Research Centre (Caucaseco). Tumaco, is a municipality situated close to the border with Ecuador, in the southwest area of Colombia with a population of 100,000 inhabitants and with Plasmodium falciparum as predominant parasite species. Buenaventura is the main port of Colombia on the Pacific coast with a population of $\sim 360,000$ habitants, with similar prevalence of both P. falciparum and Plasmodium vivax infections. Monteria is the political capital of the department of Córdoba, with a population of $\sim 300,000$ inhabitants and Tierralta, a town located about $100 \mathrm{~km}$ south of Monteria, with a population of $\sim 80,000$, with predominance of $P$. vivax infections. Patients were mostly Afro-descendants of both genders, seeking routine care for febrile episodes and were enrolled into the study after signing an informed consent form. Before the conduct of the trial, the protocol was reviewed and approved by the institutional ethics committee (CECIV, May 2011, Code 008).

\section{Study population}

Eligible participants were consecutively enrolled in the dispensaries and at the outpatient clinics of the institutions described above. Inclusion criteria were age $\geq$ one year, a documented fever or a history of fever in the previous 48 hours and a written informed assent/consent to participate in the study. No information regarding recent use of anti-malarials was collected. Study technicians enrolled study participants and collected demographic and 
clinical information electronically in the device using a structured form. After enrolment, blood was collected once from a finger prick (no follow-ups) and about three to four blood drops were used for preparation of TBS, RDTs, and a filter-paper blood spot for real-time polymerase chain reaction (PCR). The latter was performed as quality control of microscopy. Technicians working at the reference laboratory were blinded to results of both visual and device interpretation of RDTs performed at the site. Likewise, results of TBS microscopy examination and PCR (when applicable) were blinded to site technician who performed the RDTs. The processing of the RDT was performed according to manufacturer recommendations and guided by a job aid displayed on the screen of the Deki Reader device (former codename "GenZero"). The device also assisted technicians at the sites in keeping track of the incubation time for each RDT processed. Once the incubation period was completed, the study technician interpreted the RDT by visual inspection of the strip and captured the result using the touch-screen of the device. The study technician then immediately inserted the cassette into the device to perform the automated interpretation of the test. The results of the device's interpretation of the RDT were concealed at all times, and were generated in all cases after the study site technician had entered results of her/his visual interpretation of RDT test results. All results of RDTs interpretation, along with a high-resolution image of the RDT captured by the device, and the patient data collected were encrypted and automatically transmitted to a central cloud database specifically designed for the purpose of the trial. Patient management was performed according to official protocols of the national malaria control programme [21], based on the results of TBS microscopic examination.

\section{Laboratory analyses and device procedures Microscopic diagnosis}

Symptomatic patients were bled by finger prick and TBS were prepared and stained using the standard 10\% Giemsa method [22]. Slides were examined under high power objective and asexual parasites were counted against 200 white blood cells (WBCs). Smears were considered negative if the examination of 200 high power fields did not reveal asexual parasites.

\section{Malaria RDTs}

The malaria RDT used in the current study was SD Bioline Malaria Antigen Pf/Pv (Catalogue No. 05FK80, Standard Diagnostics Inc., Hagal-Dong, Korea), referred here to as RDT. This is a lateral flow immunochromatographic test that contains a membrane strip encased in a flat plastic cassette. The strip is pre-coated with two monoclonal antibodies: one specific for P. falciparum HRP2 protein and the other one specific for $P$. vivax $\mathrm{pLDH}$ enzyme.
Interpretation of the results was based first on the presence of the control line, and then on the presence of one or two lines.

\section{PCR correction}

PCR was adapted from Rougemont et al. [23], and performed in all cases of discrepant results between expert visual interpretation of RDT, device's interpretation of RDT and expert microscopy results (tie-breaker). Genomic DNA was extracted from dried filter paper spots using Chelex-resin (Biorad, Hercules, CA, USA) according to the method of Wooden et al. [24]. PCR was performed using the same set of primers and species-specific probes (Applied Biosystems, USA), with reporters and quenchers adapted to the dye channels of ABI7500 device (Applied Biosystems, USA). Duplex PCR reactions were run in parallel to detect $P$. falciparum and $P$. vivax, the $20 \mu \mathrm{l}$ reaction mix contained $2 \mu \mathrm{l}$ DNA, $1 \times$ TaqMan Master Mix (Applied Biosystems, USA), $200 \mathrm{nM}$ forward and reverse primer, $200 \mathrm{nM}$ P. falciparum probe and $100 \mathrm{nM}$ P. vivax probe. The PCR programme consisted of an initial step of $10 \mathrm{~min}$ at $95^{\circ} \mathrm{C}$ followed by 40 cycles of $10 \mathrm{sec}$ at $95^{\circ} \mathrm{C}$ and finally $60 \mathrm{sec}$ at $60^{\circ} \mathrm{C}$. Each experiment included the test sample in duplicate, non-infected DNA as negative control and a positive control with a known parasitaemia for $P$. vivax and $P$. falciparum. Parasitaemia was calculated using an average standard curve of pre-determined parasitaemia. The detection limit calculated was one parasite/ml based on the upper limit of the calibration curve at the lowest detected concentration. In addition, PCR was performed in a randomly selected subset of samples, and used in an ad hoc analysis with PCR results as gold standard $(n=274)$.

\section{The Deki Reader ${ }^{\mathrm{TM}}$ (formerly known with codename "GenZero")}

This is a mobile, ruggedized, battery-operated device (Figure 2), manufactured by Fio Corporation, (Toronto, Canada), designed to perform the following functions: 1) automated interpretation of RDTs by means of image analysis software; 2) digital data capture, by means of a touch-screen and a simple user interface software; and, 3) transmission in real-time (RT) of all data to an accessible central database, using local mobile phone network. Transmitted data included: processed RDT image, diagnostic event data collected, user and device interpretation of test results, geo-positioning of the device, and date and time stamp. The principal investigator (PI) and study coordinators had permanent access to the database via the internet portal especially designed for the study.

\section{Training on the operation of Deki Reader}

Seven laboratory microbiologists with five years of university education and former training on malaria microscopy were engaged to operate the Deki Reader and to fill 




Figure 2 Deki Reader device.

up all required documentation for the study. These laboratory personnel were trained in a two-day workshop on all study procedures, the operation of RDTs assembled to a Deki Reader, as well as on the collection of patients' information, including the informed consent form.

\section{Statistical methods}

The prevalence of malaria cases ( $P$. falciparum and $P$. vivax) at the study areas was estimated to be 10 to $15 \%, P$. vivax being responsible for $\geq 75 \%$ of the malaria cases. Based on this prevalence, the sample size was estimated to be between 1,800 to 2,000 subjects, under the assumption that it would provide approximately 120 to 225 positive results for $P$. vivax and 40 to 75 positive results for $P$. falciparum. Assuming that the sensitivity and specificity of the interpretation of RDT is the same as the human-eye interpretation, this sample size would provide $95 \%$ confidence intervals within $5 \%$ of the point estimate. Results of RDT were interpreted by both visual examination and by the Deki Reader device.

Data were obtained from the source documents at each of the participant malaria sites and was compared with the information collected through devices and transmitted to the cloud database. Data collected were monitored according to U S Food and Drug Administration (FDA) regulations and Good Clinical Practice (GCP) guidelines.

There were three outcomes of interest for the purpose of the analysis in the current trial: a) microscopy results: performed on TBS by laboratory technician at reference laboratory and entered manually into the dataset; $\mathrm{b}$ ) visual interpretation of RDT: performed by laboratory technician at the site following visual inspection of the RDT at the appropriate time, and entered into the Deki Reader device; and, c) device interpretation of RDT: performed by the device, which was automatically collected and transmitted to the database by the device. Data collected at the cloud database were reconciled to data collected in the internal memory card of each Deki Reader device to ensure that all data points were correctly transmitted to the cloud database. Data analysis was performed using JMP Version 8.0.2 and R Version 2.12.1 (SAS Institute Inc. NC, USA). The primary analysis was conducted using microscopy corrected by PCR as gold standard to determine diagnostic performance characteristics $[25,26]$ (sensitivity, specificity, negative (NPV) and positive (PPV) predictive values, and overall diagnostic accuracy) of RDT interpretation by the Deki Reader and by visual interpretation. Secondly, microscopy and PCR were used as gold standards. Correspondent 95\% confidence intervals were constructed using the Wilson score method. The percentage of agreement in the interpretation of RDTs between the device and visual (human-eye) done by the expert laboratory technicians at the sites was calculated as percentage of negative agreement, percentage of positive agreement and overall percentage of agreement. Two-sided $95 \%$ confidence intervals were constructed using the Wilson score method. Fisher's exact test was used to compare differences between device and visual interpretation and $\mathrm{P}$ value $<0.05$ was considered statistically significant.

\section{Results}

Between August 2011 and January 2012, a total of 2,191 subjects were screened. However, the final analysis included 1,807 samples, after 384 (17.5\%) were excluded due to lack 
of compliance with the protocol: 238 (62\%) were samples collected in a centre where RDT processing was performed in major violation of RDT manufacturer's recommendation; 78 (20\%) patient samples due to incomplete written informed consent process, and 68 (18\%) due to RDT processing by non-study authorized personnel (Figure 3).

The main demographic features of the cohort are described in Table 1 . The proportion of female participants was $54.3 \%$, and the population was predominantly adults (76.4\%) between 17 and 65 years of age. The prevalence of microscopically confirmed malaria cases among the recruited patients was $7.58 \%$ (33 cases of positive $P$. falciparum infection and 100 cases of $P$. vivax). Positive cases varied among the sites between 1.89 and $5.84 \%$ for P. faciparum, and between 1.52 and $10.54 \%$ for P. vivax, which is in agreement to the local known epidemiology concerning distribution of Plasmodium species infections [27]. Prevalence of $P$. falciparum infections was higher in the sites of Tumaco and Buenaventura (25 P. falciparum and 30 P. vivax infections), while $P$. vivax infection was higher in Tierralta (eight P. falciparum and $70 P$. vivax infections).

Of the 2,191 samples, in 37 (1.7\%) no primary or secondary outcomes (regarding results of the interpretation of RDTs), either visual or by the device were found in the dataset, most likely due to software malfunction, and these patients were not included in the analysis. Therefore, the final analysis dataset consisted of 1,770 samples (Figure 3).
Table 1 Demographics of study population

\begin{tabular}{ll}
\hline Enrolled $(\mathbf{N}=\mathbf{1 , 8 0 7})$ & \\
\hline Gender & $982(54.3 \%)$ \\
Female & $825(45.7 \%)$ \\
Male & \\
Age (years) & $30.74(17.05)$ \\
$\quad$ Mean (SD) & 27.92 \\
Median & {$[0.2,88]$} \\
Range & \\
Age categories (years) & $16(0.9 \%)$ \\
$<1$ & $341(18.9 \%)$ \\
$\geq 1$ to $<17$ & $1,380(76.4 \%)$ \\
$\geq 17$ to $<65$ & $70(3.8 \%)$ \\
$\geq 65$ &
\end{tabular}

\section{Comparison between diagnostic performance} characteristics of the visual interpretation and Deki Reader interpretation of RDTs

The percentage agreement between visual interpretation and Deki Reader interpretation of RDTs for $P$. falciparum was $95.7 \%$ (95\% CI 85.5-98.8) when a positive result was obtained, 99.1\% (95\% CI 98.5-99.4) when a negative result was obtained, and an overall percentage agreement of 99.0\% (95\% CI 98.4-99.4) (Table 2). The percentage interpretation agreement between the Deki Reader and visual for $P$. vivax was 98.9\% (95\% CI 94.0-99.5), when a negative result was obtained; $99.1 \%$ (95\% CI 97.8-99.0)

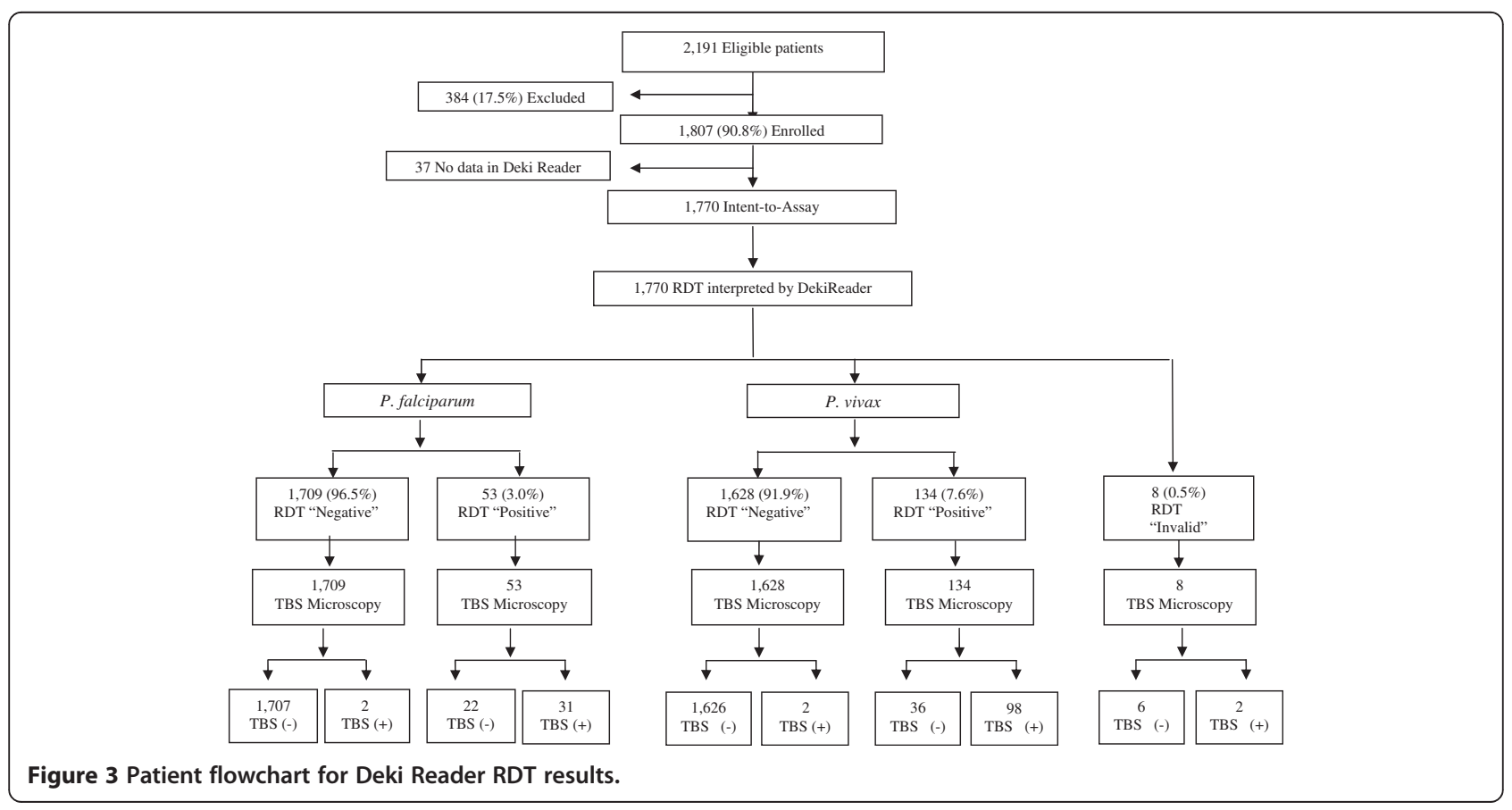


Table 2 Comparison of performance between Deki Reader and visual interpretation $(n=1,762)$ of RDTs

\begin{tabular}{lccc}
\hline Parasite species & $\begin{array}{c}\text { Negative \% } \\
\text { agreement } \\
(\mathbf{9 5 \%} \mathbf{C l})\end{array}$ & $\begin{array}{c}\text { Positive \% } \\
\text { agreement } \\
(\mathbf{9 5 \%} \mathbf{C l})\end{array}$ & $\begin{array}{c}\text { Overall \% } \\
\text { agreement } \\
(\mathbf{9 5 \%} \mathbf{C l})\end{array}$ \\
\hline P. falciparum & $99.1(98.5-99.4)$ & $95.7(85.5-98.8)$ & $99.0(98.4-99.4)$ \\
P. vivax & $98.9(94.0-99.5)$ & $99.1(97.8-99.0)$ & $98.5(97.9-99.0)$ \\
\hline
\end{tabular}

when a positive result was obtained; and an overall agreement of 98.5\% (95\% CI 97.9-99.0). There was no statistically significant difference in the sensitivity or in the specificity between the two methods of interpretation for any of the Plasmodium species.

\section{Discordant results between Deki Reader and visual interpretation of RDTs}

From a total of 30 discrepant test results, discordant results were obtained in 26 RDTs (1.47\%) for either $P$. falciparum or $P$. vivax results and two showed discordant results for both Plasmodium species. In 28/30 (93\%) discrepant interpretations, the visual inspection failed to detect a line, but the device reported a "positive" result. In the remaining two cases, it was the opposite. In ten (33\%) of the 30 discrepant results, a closer review of the images of the RDTs revealed the presence of artefacts and bizarre smearing of the test strip that could have caused the software in the device to interpret as presence of a line. The presence of these unusual patterns in the flow of the sample along the diagnostic strip renders the test inconclusive and therefore should have resulted in processing a new RDT. In an additional seven cases, including the two where the visual inspection reported the presence of a line, the discrepancy seems to be originated from clerical mistakes by either registering a negative result when clearly visible lines were present in the RDT, registering a positive result when no lines were visible and no parasites were reported either by microscopy or PCR, or by mistakes in the identification of the tests. In an additional three cases, results of microscopy and/or PCR confirmed the presence of parasites and therefore were true positive cases (device interpretation was correct). In the remaining ten cases, all of which the device interpreted as positive with line intensity values very close to the cut-off value, both microscopy and PCR failed to detect presence of parasites and therefore correspond to false positive results $(0.56 \%)$ (device interpretation was incorrect).

\section{Sensitivity and specificity of Deki Reader and visual interpretation of RDTs}

Of the 1,770 samples tested, 94 were positive for Plasmodium by both diagnostic methods (TBS and RDT), 1,525 were negative by both diagnostic tests and 132 had discordant results. Of these discordant samples, 70
(4\%) were positive only by RDT, 43 (2\%) were positive only by TBS and 19 (1\%) had discordant parasite species results. This discrepancies were solved using PCR as a tie-breaker, finding that 24 (34\%) of the 70 RDT-positive samples were positive, 39 (90\%) of the 43 TBS-positive samples were negative and $74 \%(14 / 19)$ the samples with species discrepancies had PCR results that were in line with RDT results.

\section{TBS microscopy as gold standard}

When standard TBS microscopy results were compared to interpretation of RDT, the Deki Reader had a sensitivity of $33.3 \%$ (95\% CI, 21.4-47.6) and a specificity of 98.1\% (95\% CI 97.3-98.7) for detection of P. falciparum infections, and sensitivity of $72.1 \%$ (95\% CI, 62.5-80.5) and a specificity of $96.5 \%$ (95\% CI 95.6-97.4) for detection of P. vivax infections. The correspondent sensitivity of expert visual interpretation of RDTs was 35.1\% (95\% CI, 22.9-48.9) and specificity of 98.4\% (95\% CI 97.7-98.9) for detection of P. falciparum infections, and sensitivity of $71.8 \%$ (95\% CI, 62.1-80.3) and a specificity of $97.4 \%$ (95\% CI 96.5-98.0) for detection of $P$. vivax infections (Table 3 ).

\section{TBS microscopy corrected by PCR as gold standard}

When compared to microscopy corrected by PCR, the Deki Reader had a sensitivity of $93.9 \%$ (95\% CI 69.7-95.2) and a specificity of 98.7\% (95\% CI 98.5-99.4) for detection of $P$. falciparum infections, and sensitivity of $98.0 \%$ (95\% CI 90.3-98.9) and a specificity of 97.9\% (95\% CI 97.1-98.5) for detection of $P$. vivax infections (Table 4). The sensitivity and specificity of expert visual interpretation of RDTs were identical to the Deki Reader for detection of $P$. falciparum infections, and for detection of $P$. vivax infections the sensitivity was $87.8 \%$ (95\% CI 86.4-97.2) and the specificity 99.0\% (100\% CI 98.0-99.14). Finally, the performance of microscopy was $60.6 \%$ (95\% CI 44.9-78.5) and 98.0\% (95\% CI 97.3-98.6) for sensitivity and specificity against P. falciparum; and 75.0\% (95\% CI 66.0-83.4) and 98.4\% (95\% CI, 97.7-99.0) for sensitivity and specificity against $P$. vivax (Table 4). The diagnostic performance of visual and Deki Reader interpretation of RDTs was virtually identical, and showed a higher sensitivity than microscopy for both species for all the gold standards used.

Table 3 Deki Reader diagnostic performance against microscopy

\begin{tabular}{lcccccc}
\hline Microscopy & & $\begin{array}{c}\text { Sensibility } \\
\text { (\%) }\end{array}$ & $\begin{array}{c}\text { Specificity } \\
\text { (\%) }\end{array}$ & $\begin{array}{c}\text { PPV } \\
\text { (\%) }\end{array}$ & $\begin{array}{c}\text { NPV } \\
\text { (\%) }\end{array}$ & P-Value* $^{*}$ \\
\hline P.vivax & Visual & 71.8 & 97.4 & 62.7 & 98.3 & 0.876 \\
& Device & 72.1 & 96.6 & 56.0 & 98.3 & \\
\multirow{2}{*}{$\begin{array}{l}\text { P.falciparum } \\
\end{array}$} & Visual & 35.1 & 98.4 & 41.7 & 97.9 & 0.843 \\
& Device & 33.3 & 98.1 & 35.9 & 97.8 & \\
\hline
\end{tabular}

*Fisher's exact test for sensibility. 
Table 4 Deki Reader diagnostic performance against microscopy corrected by PCR

\begin{tabular}{lcccccc}
\hline $\begin{array}{l}\text { Microscopy } \\
\text { corrected by PCR }\end{array}$ & $\begin{array}{c}\text { Sensibility } \\
\text { (\%) }\end{array}$ & $\begin{array}{c}\text { Specificity } \\
\text { (\%) }\end{array}$ & $\begin{array}{c}\text { PPV } \\
\text { (\%) }\end{array}$ & $\begin{array}{c}\text { NPV } \\
\text { (\%) }\end{array}$ & P-Value* \\
\hline P. vivax & Visual & 94.0 & 98.6 & 80.5 & 99.6 & 0.149 \\
& Device & 98.0 & 97.8 & 73.1 & 99.8 & \\
\multirow{2}{*}{$\begin{array}{l}\text { P. falciparum } \\
\end{array}$} & Visual & 87.8 & 99.0 & 62.5 & 99.7 & 0.392 \\
& Device & 93.9 & 98.7 & 62.5 & 99.7 & \\
\hline
\end{tabular}

*Fisher's exact test for sensibility.

\section{PCR as quality control for microscopy}

When compared to PCR results performed in a subset of 274 randomly selected samples the performance of microscopy was $54.6 \%$ (95\% CI 23.4-83.3) and $98.9 \%$ (95\% CI 96.7-99.8) for sensitivity and specificity against P. falciparum; and 68.0\% (95\% CI 46.5-85.0) and $98.8 \%$ (95\% CI 96.5-99.75) for sensitivity and specificity against P. vivax (Table 5).

\section{Discussion}

Early and adequate malaria diagnosis is critical to opportune and effective treatment to prevent clinical complications and death. Among the malaria diagnostic tools currently available, microscopic examination of TBS remains the gold standard for defining malaria infection, not only because of the relative ease and low cost, but also for its ability to provide a quantitative result. Malaria RDTs are particularly useful in remote areas of difficult access and lack of basic infrastructure required in a microscopy post. The interpretation of two test lines contained in the RDT may be challenging from the point of view of personnel with low level of education, training and supervision (i e, community health workers) located in remote rural areas. Although the SD Bioline Malaria Antigen Pf/Pv RDT imposes this difficulty, it was decided to use this RDT in combination with the Deki Reader due to the known presence of both parasite species in the study areas. A previous study conducted in rural health facilities in Tanzania demonstrated that interpretation of SD Bioline Malaria Ag Pf/ Pan RDT by Deki Reader is substantially equivalent to that of visual interpretation as performed by laboratory technicians with vast experience in RDT processing and interpretation [28], suggesting that a device such as the Deki Reader can be used in routine care for interpretation of RDT with high-quality diagnostic results at POC.

In the present study, the performance of the Deki Reader interpreting SD Bioline Pf/Pv RDTs was at least as good as

\section{Table 5 Quality control of microscopy using PCR on a} subset of samples

\begin{tabular}{lcccc}
\hline PCR & Sensibility (\%) & Specificity (\%) & PPV (\%) & NPV (\%) \\
\hline P. vivax & 68.0 & 98.8 & 85.0 & 96.9 \\
P. falciparum & 60.6 & 98.0 & 66.7 & 98.1 \\
\hline
\end{tabular}

the visual interpretation by experts, with sensitivity, specificity, positive and negative predictive value similar to visual interpretation of the same RDT (Tables 2 and 3). There was no statistically significant difference between the diagnostic accuracy of the Deki Reader and the visual interpretation of RDTs (Fisher's exact test).

Important differences in the accuracy of the Deki Reader were identified when microscopy was used as the gold standard for malaria diagnosis. Most of the RDT-positive/ microscopy-negative discordance is likely due to reduced specificity as result of the persistent antigenaemia of P. falciparum for approximately two weeks after parasite clearance [29] and most of microscopy-positive/RDTnegative cases might have originated in deficiencies in the microscopy test.

Similar findings to the present study with respect to comparative performance of microscopy versus RDTs have been reported previously [30-35], and the sensitivity and specificity of the RDTs with this reference test were in agreement with previous reports, including the third evaluation round of FIND [12]. In the same way, other studies using microscopy corrected by PCR suggested that RDTs may actually be more sensitive than expert slide reading [36]. However, RDTs are not able to replace microscopy as the gold standard for malaria diagnosis, they can be used as complementary technique in situation where experienced personnel are not available, in order to perform a rapid diagnosis, which can a few hours later be confirmed or corrected by microscopy or PCR.

The areas selected for this study, although distant from main cities of Colombia, benefit from electricity, basic laboratory facilities and are reachable by road. They all had sufficient conditions for proper storage of the test kits. Deki Reader batteries could be recharged easily when needed and communication infrastructure for mobile and land telephone were both available.

TBS microscopy presented serious limitations, with factors such as variability in techniques of blood film preparation, staining, reading standards, and most importantly, highly dependent on the level of expertise of microscopists. In the present study, laboratory technicians had training and experience above the standard level of field microscopist. Despite their university training, the significant loss of information for the final analysis $(384=17.5 \%)$, most of which $(238=10.8 \%)$ presented major violation of RDT manufacturer's recommendation, indicated that although constant monitoring was available, the two-day training may have been insufficient. The PI and study coordinators had permanent access to the database via internet portal especially designed for the study and were able to monitor the progress of the study and to review images of the RDTs which allowed them to redirect training efforts to the areas where images suggested RDT mishandling. Device users in the field were able to perform the data collection 
using the touch screen and processing and interpretation of RDTs without major problems. Likely, due to software malfunction early in the course of the study, data from 37 samples $(1.7 \%)$ were not recorded in the device nor transmitted to the database.

Although some places in remote endemic regions do not yet have reliable mobile phone connectivity, the device saves all data which can be transmitted later under good connections to mobile networks. The data and images displayed in the portal created for this study allowed to follow up closely the performance of field operators. It has also demonstrated the potential to be greatly beneficial to health programme managers by providing a tool to perform remote QA monitoring of field activities. The ability to assist in POC diagnosis, and simultaneously capture and make immediately available pivotal epidemiological data at source of origin makes this a very attractive tool to enhance epidemiological surveillance, strengthen health care systems, and contribute to global mapping efforts of malaria and other infectious diseases [37-39]. The information collected, along with the correspondent RDT image, was sent immediately from remote areas to a database, being part of the Fio Cloud services with $100 \%$ fidelity.

Although the function of the Deki Reader is to interpret the presence or absence of a test line signal, independent of the analyses that are finally deposited on it, these results could not be extrapolated to other RDTs. Important differences in RDT clinical diagnostic performance have been well documented, and therefore the results presented here are only applicable to SD Bioline Malaria Antigen Pf/Pv RDT. Nevertheless, the performance of the Reader should be independent to the underlying immunochemistry and thus should provide similar performance characteristics for other RDTs using the same immunochromatographic test.

\section{Conclusions}

The diagnostic performance of the Deki Reader was comparable to visual interpretation of RDTs (concordance rate $>98 \%$ ) for both malaria species. Providing standardized automated interpretation of RDTs at POC in remote areas, in addition to almost real-time reporting of cases and enabling quality control would greatly benefit large-scale implementation of RDT-based malaria diagnostic programmes.

\section{Competing interests}

The authors declare that they have no competing interests. SF and MC are employees of Fio Corporation.

\section{Authors' contributions}

$\mathrm{SH}, \mathrm{MA}-\mathrm{H}$ and SF conceived and designed the study. SH, MA-H, AV and SF wrote the manuscript. MC and JPQ collected and monitored the data, and provided comments on the manuscript. All authors read and approved the final manuscript.

\section{Acknowledgements}

We would like to thank all patients who consented to participate in this study, and all laboratory technicians. Part of this study was sponsored and conducted in sites corresponding to Centro Latino Americano de Investigación en Malaria (CLAIM) sponsored by NIAID/ICEMR (1U19AI089702) and Colciencias (360-2011).

\section{Author details}

${ }^{1}$ Caucaseco Scientific Research Center, Cali, Colombia. ${ }^{2}$ Latin American Center for Malaria Research, Cali, Colombia. ${ }^{3}$ Faculty of Health, Universidad del Valle, Cali, Colombia. ${ }^{4}$ Fio Corporation, Toronto, Canada.

Received: 24 September 2013 Accepted: 2 March 2014

Published: 10 March 2014

\section{References}

1. World Health Organization: 10 facts on malaria. In http://www.who.int/ features/factfiles/malaria/en

2. WHO: Guidelines for the Treatment of Malaria. World Health Organization; 2006.

3. Merwyn S, Gopalan N, Singh AK, Rai GP, Agarwal GS: Monoclonal antibodies against recombinant histidine-rich protein 2 of Plasmodium falciparum and their use in malaria diagnosis. Hybridoma 2011, 30:519-524.

4. Kattenberg JH, Versteeg I, Migchelsen SJ, González IJ, Perkins MD, Mens PF, Schallig HD: New developments in malaria diagnostics: monoclonal antibodies against Plasmodium dihydrofolate reductase-thymidylate synthase, heme detoxification protein and glutamate rich protein. In mAbs Landes Bioscience; 2012:120-126.

5. Schellenberg D: A research agenda for malaria eradication: diagnoses and diagnostics. PLoS Med 2011, 8:e1000396.

6. Pöschl B, Waneesorn J, Thekisoe O, Chutipongvivate S, Panagiotis K: Comparative diagnosis of malaria infections by microscopy, nested PCR, and LAMP in northern Thailand. Am J Trop Med Hyg 2010, 83:56.

7. Mtove G, Hendriksen I, Amos B, Mrema H, Mandia V, Manjurano A, Muro F, Sykes A, Hildenwall $H$, Whitty C: Treatment guided by rapid diagnostic tests for malaria in Tanzanian children: safety and alternative bacterial diagnoses. Malar J 2011, 10:290.

8. Masanja Ml, McMorrow M, Kahigwa E, Kachur SP, McElroy PD: Health workers' use of malaria rapid diagnostic tests (RDTs) to guide clinical decision making in rural dispensaries, Tanzania. Am J Trop Med Hyg 2010, 83:1238-1241.

9. Njama-Meya D, Clark TD, Nzarubara B, Staedke S, Kamya MR, Dorsey G: Treatment of malaria restricted to laboratory-confirmed cases: a prospective cohort study in Ugandan children. Malar J 2007, 6:7.

10. D'Acremont V, Malila A, Swai N, Tillya R, Kahama-Maro J, Lengeler C, Genton $B$ : Withholding antimalarials in febrile children who have a negative result for a rapid diagnostic test. Clin Infect Dis 2010, 51:506-511.

11. WHO: Good Practices for Selecting and Procuring Rapid Diagnostic Tests for Malaria. 2011.

12. WHO: Malaria Rapid Diagnostic Test Performance; Results of WHO product testing of malaria RDTs: Round 3 (2011). 2011. Geneva: World Health Organization; 2012.

13. Rennie W, Phetsouvanh R, Lupisan S, Vanisaveth V, Hongvanthong B, Phompida S, Alday P, Fulache M, Lumagui R, Jorgensen P: Minimising human error in malaria rapid diagnosis: clarity of written instructions and health worker performance. Trans R Soc Trop Med Hyg 2007, 101:9-18.

14. Harvey SA, Jennings L, Chinyama M, Masaninga F, Mulholland K, Bell DR: Improving community health worker use of malaria rapid diagnostic tests in Zambia: package instructions, job aid and job aid-plus-training. Malar J 2008, 7:160.

15. WHO: Universal access to malaria diagnostic testing. 2011.

16. McMorrow ML, Masanja MI, Kahigwa E, Abdulla SM, Kachur SP: Quality assurance of rapid diagnostic tests for malaria in routine patient care in rural Tanzania. Am J Trop Med Hyg 2010, 82:151-155.

17. McMorrow ML, Masanja MI, Abdulla SM, Kahigwa E, Kachur SP: Challenges in routine implementation and quality control of rapid diagnostic tests for malaria-Rufiji District, Tanzania. Am J Trop Med Hyg 2008, 79:385-390.

18. Mukadi P, Leion V, Lukuka A, Mbatshi J, Otshudiema J, Muyembe J-J, Gillet $P$, Jacobs J: SMS based external quality assessment of reading and interpretation of malaria rapid diagnostic tests: Preliminary results among more than 2000 end-users in the Democratic Republic of the Congo. Malar J 2012, 11:P65.

19. Learmonth KM, MCPhee DA, Jardine DK, Walker SK, Aye T-T, Dax EM: Assessing proficiency of interpretation of rapid human immunodeficiency virus assays 
in nonlaboratory settings: ensuring quality of testing. J Clin Microbiol 2008, 46:1692-1697.

20. Chiu Y-HC, Ong J, Walker S, Kumalawati J, Gartinah T, McPhee DA, Dax EM: Photographed rapid HIV test results pilot novel quality assessment and training schemes. PLOS ONE 2011, 6:e18294.

21. Protocolo de vigilancia en salud pública de la malaria. In http://www.ins.gov co/temas-de-interes/Documentacin\%20Malaria/01\%20Protocolo\%20Malaria.pdf.

22. Barber $\mathrm{M}, \mathrm{Komp} \mathrm{W}$ : Method of preparing and examining thick films for the diagnosis of malaria. Public Health Rep 1896-1970, 1929:2330-2341.

23. Rougemont M, Van Saanen M, Sahli R, Hinrikson HP, Bille J, Jaton K: Detection of four Plasmodium species in blood from humans by $18 \mathrm{~S}$ rRNA gene subunit-based and species-specific real-time PCR assays. J Clin Microbiol 2004, 42:5636-5643.

24. Wooden J, Kyes S, Sibley C: PCR and strain identification in Plasmodium falciparum. Parasitol Today 1993, 9:303-305.

25. Eibach D, Traore B, Bouchrik M, Coulibaly B, Coulibaly N, Siby F, Bonnot G, Bienvenu A-L, Picot S: Evaluation of the malaria rapid diagnostic test VIKIA malaria Ag Pf/PanTM in endemic and non-endemic settings. Malar J 2013, 12:188.

26. Hopkins H, Bebell L, Kambale W, Dokomajilar C, Rosenthal PJ, Dorsey G: Rapid diagnostic tests for malaria at sites of varying transmission intensity in Uganda. J Infect Dis 2008, 197:510-518.

27. Semanal IBE: Estadísticas del sistema de vigilancia en salud pública-SIVIGILA. In Casos Totales en la Semana Epidemiológica. 2009:52.

28. Shekalaghe S, Cancino M, Mavere C, Juma O, Mohammed A, Abdulla S, Ferro S: Clinical performance of an automated reader in interpreting malaria rapid diagnostic tests in Tanzania. Malar J 2013, 12:141.

29. Mayxay M, Pukrittayakamee S, Chotivanich K, Looareesuwan S, White NJ: Persistence of Plasmodium falciparum HRP-2 in successfully treated acute falciparum malaria. Trans R Soc Trop Med Hyg 2001, 95:179-182.

30. Kahama-Maro J, D'Acremont V, Mtasiwa D, Genton B, Lengeler C: Low quality of routine microscopy for malaria at different levels of the health system in Dar es Salaam. Malar J 2011, 10:332.

31. Baliraine FN, Afrane YA, Amenya DA, Bonizzoni M, Menge DM, Zhou G, Zhong D, Vardo-Zalik AM, Githeko AK, Yan G: High prevalence of asymptomatic Plasmodium falciparum infections in a highland area of western Kenya: a cohort study. J Infect Dis 2009, 200:66-74.

32. Okell LC, Ghani AC, Lyons E, Drakeley CJ: Submicroscopic infection in Plasmodium falciparum-endemic populations: a systematic review and meta-analysis. J Infect Dis 2009, 200:1509-1517.

33. De Oliveira AM, Skarbinski J, Ouma PO, Kariuki S, Barnwell JW, Otieno K, Onyona P, Causer LM, Laserson KF, Akhwale WS: Performance of malaria rapid diagnostic tests as part of routine malaria case management in Kenya. Am J Trop Med Hyg 2009, 80:470-474.

34. Mboera L, Fanello C, Malima R, Talbert A, Fogliati P, Bobbio F, Molteni F: Comparison of the Paracheck-Pf $f^{\oplus}$ test with microscopy, for the confirmation of Plasmodium falciparum malaria in Tanzania. Ann Trop Med Parasitol 2006, 100:115-122.

35. Zurovac D, Larson B, Akhwale W, Snow R: The financial and clinical implications of adult malaria diagnosis using microscopy in Kenya. Trop Med Int Health 2006, 11:1185-1194.

36. Bell DR, Wilson DW, Martin LB: False-positive results of a Plasmodium falciparum histidine-rich protein 2-detecting malaria rapid diagnostic test due to high sensitivity in a community with fluctuating low parasite density. Am J Trop Med Hyg 2005, 73:199-203.

37. Bloland P, Simone P, Burkholder B, Slutsker L, De Cock KM: The role of public health institutions in global health system strengthening efforts: the US CDC's perspective. PLoS Med 2012, 9:e1001199.

38. Hay SI, Battle KE, Pigott DM, Smith DL, Moyes CL, Bhatt S, Brownstein JS, Collier N, Myers MF, George DB: Global mapping of infectious disease. Philos Trans R Soc Lond B Biol Sci 2013, 368:20120250.

39. Afrane YA, Zhou G, Githeko AK, Yan G: Utility of health facility-based malaria data for malaria surveillance. PLOS ONE 2013, 8:e54305.

\section{doi:10.1186/1475-2875-13-87}

Cite this article as: Herrera et al.: Field evaluation of an automated RDT reader and data management device for Plasmodium falciparum/ Plasmodium vivax malaria in endemic areas of Colombia. Malaria Journal 2014 13:87.

\section{Submit your next manuscript to BioMed Central and take full advantage of:}

- Convenient online submission

- Thorough peer review

- No space constraints or color figure charges

- Immediate publication on acceptance

- Inclusion in PubMed, CAS, Scopus and Google Scholar

- Research which is freely available for redistribution 